\title{
High-resolution CO Survey of Virgo Spirals at Nobeyama - Massive Cores inferred from Central Rotation Curves
}

\author{
Y. Sofue ${ }^{1}$, H. Nakanishi ${ }^{1}$, S. Onodera ${ }^{1}$, J. Koda ${ }^{2,3}, \&$ K. Kohno ${ }^{1}$ \\ 1 Inst. Astro., Univ. Tokyo, Japan; ${ }^{2}$ Nobeyama Radio Observatory, \\ NAOJ, Japan; ${ }^{3}$ Caltech, USA
}

\begin{abstract}
We present the results of a high-resolution CO-line survey using the Nobeyama Millimeter-wave Array at high-angular $\left(\sim 2-3^{\prime \prime}\right)$ and high spectral resolutions for the 15 CO-richest Virgo spirals. We derived exact rotation curves using position-velocity diagrams by applying the iteration method. The obtained RCs rise steeply in the central $100 \mathrm{pc}$. Surface-mass-density distributions by direct deconvolution of the RCs show massive cores of $\sim 10^{9} M_{\odot}$ within $100 \mathrm{pc}$, suggesting the existence of DM cusps in the centres. Five galaxies were found to nest single-peaked extremely high-density molecular cores, where star formation is currently suppressed by the high differential rotation. We show that the deeper is the gravitational potential, the higher is the central gas density.
\end{abstract}

\section{Introduction}

We performed a high-resolution ${ }^{12} \mathrm{CO}(J=1-0)$-line survey using the Nobeyama Millimeter-wave Array (NMA) in 1999 December through 2002 April (Sofue et al. 2003a). The objects were selected from Virgo cluster members, while considering the CO richness from the single-dish flux, mild inclination, and lack of strong tidal perturbations. The central $1^{\prime}$ regions $(\sim 4.7 \mathrm{kpc})$ of 15 spiral galaxies were observed at spatial and angular resolutions of $2-5^{\prime \prime}$ and $10 \mathrm{~km} \mathrm{~s}^{-1}$ (Fig. 1). The objects lie near the Virgo cluster centre at the same distance of 16.1 Mpc, advantageous for comparisons among individual galaxies.

\section{High-accuracy Central Rotation Curves and Massive Cores - DM cusp?}

We applied a new iteration method to the obtained position-velocity diagrams (PVD) to derive rotation curves (RCs), which reproduce the observed PVDs and is particularly accurate in the central regions (Fig. 2, 3). The high-accuracy RCs generally show a steep rise in the central $\sim 100$ regions, followed by nearly flat rotation in the bulge and disk (Sofue et al. 2003b).

We deconvolved the RCs to calculate the surface-mass density (SMD: Fig. 4). The SMD distributions show central massive cores with peak SMD of $10^{4}-10^{5} M_{\odot} \mathrm{pc}^{-2}$ and a total mass within $100 \mathrm{pc}$ radius of the order of $10^{9} M_{\odot}$. The central SMD and the masses of bulge and massive cores are are 

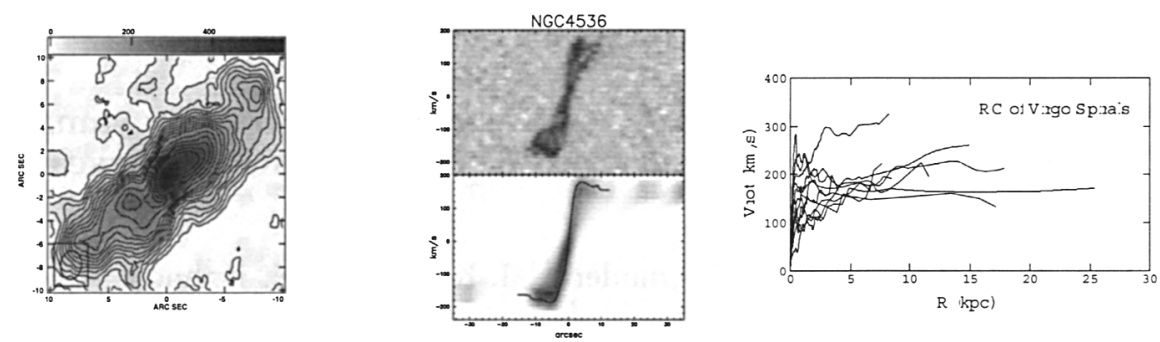

Fig. 1. (left) Example of the Virgo CO atlas: NGC 4536 showing a single peak. Fig. 2. (centre) PVD and RC after iteration for NGC 4536.

Fig. 3. (right) Rotation curves of Virgo spirals.
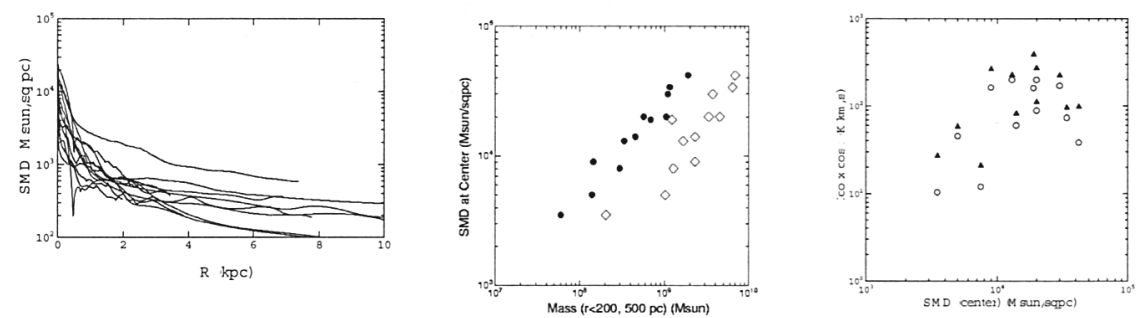

Fig. 4. (left) Surface mass density (SMD) distributions.

Fig. 5. (centre) Central SMD vs bulge (diamond) and core (circle) masses.

Fig. 6. (right) Central CO intensity vs central SMD.

well correlated with each other (Fig. 5). The massive cores of $\sim 10^{9} M_{\odot}$ in the central $100 \mathrm{pc}$ are found to be universal, indicating dark matter cusps.

\section{3. "Single-Peak" High-density Molecular Cores in Deep Potentials}

A correlation analysis shows that the central CO-line intensity is positively correlated with the central SMD (Fig. 6), indicating that the deeper is the gravitational potential, the higher is the molecular gas concentration, regardless of the morphological type (Sofue et al. 2003b).

Most of the galaxies show a high concentration of gas in the central few kpc region. Particularly, five galaxies show "single-peak" molecular cores with extremely high CO intensities (Fig. 1), while only one galaxy shows "twinpeaks". The single-peak type is more general than twin peaks for the central molecular gas morphology.

\section{References}

Sofue, Y., Koda, J., Nakanishi, H., et al. 2003a, PASJ, 55, 17

Sofue, Y., Koda, J., Nakanishi, H., \& Onodera, S. 2003b, PASJ, 55, 59 